\title{
Status of jet cross sections to NNLO
}

\author{
Stefan Weinzierl ${ }^{\mathrm{a}}$ \\ ${ }^{\text {a} I n s t i t u t ~ f u ̈ r ~ P h y s i k, ~ U n i v e r s i t a ̈ t ~ M a i n z, ~ D ~-~} 55099$ Mainz, Germany
}

I review the state-of-the-art for fully differential numerical NNLO programs. Topics which are covered include the calculation of two-loop amplitudes, multiple polylogarithms, cancellation of infra-red divergences at NNLO and the efficient generation of the phase space. Numerical results for $e^{+} e^{-} \rightarrow 2$ jets are also discussed.

\section{INTRODUCTION}

In the near future the LHC experiments will provide us with a large sample of multi-particle final states. In order to extract information from this data, precise theoretical calculations are necessary. This implies to extend perturbative calculations for selected processes from next-toleading order (NLO) to next-to-next-to-leading order (NNLO) in the perturbative expansion in the strong coupling constant. Due to a large variety of interesting jet observables it is desirable not to perform this calculation for a specific observable, but to set up a computer program, which yields predictions for any infra-red safe observable relevant to the process under consideration.

The past years have witnessed a tremendous progress in techniques for the computation of two-loop integrals and in the calculation of twoloop amplitudes [1]. In addition, several options for the cancellation of infra-red divergences have been discussed [2-12]. Among those, the subtraction method - well-known from NLO computations [13-17] - and sector decomposition [18] are the most promising candidates. First numerical results have become available for the processes $e^{+} e^{-} \rightarrow 2$ jets and $H \rightarrow \gamma \gamma[19-22]$.

\section{TWO-LOOP AMPLITUDES}

The master formula to calculate an observable at an collider with no initial-state hadrons (e.g. an electron-positron collider) is given by

$$
\left\langle\mathcal{O}^{(j)}\right\rangle=\frac{1}{2 K(s)} \frac{1}{\left(2 J_{1}+1\right)} \frac{1}{\left(2 J_{2}+1\right)}
$$

$$
\sum_{n} \int d \phi_{n-2} \mathcal{O}_{n}^{(j)}\left(p_{1}, \ldots, p_{n}\right) \sum_{\text {helicity }}\left|\mathcal{A}_{n}\right|^{2}
$$

where $p_{1}$ and $p_{2}$ are the momenta of the initialstate particles, $2 K(s)=2 s$ is the flux factor and $s=\left(p_{1}+p_{2}\right)^{2}$ is the center-of-mass energy squared. The factors $1 /\left(2 J_{1}+1\right)$ and $1 /\left(2 J_{2}+1\right)$ correspond to an averaging over the initial helicities. $d \phi_{n-2}$ is the invariant phase space measure for $(n-2)$ final state particles and $\mathcal{O}_{n}^{(j)}\left(p_{1}, \ldots, p_{n}\right)$ is the observable, evaluated with an $n$-parton configuration. The index $j$ indicates that the leading order contribution depends on $j$ partons. The observable has to be infra-red safe, in particular this implies that we must have in single unresolved limits

$\mathcal{O}_{n+1}^{(j)}\left(p_{1}, \ldots, p_{n+1}\right) \rightarrow \mathcal{O}_{n}^{(j)}\left(p_{1}^{\prime}, \ldots, p_{n}^{\prime}\right)$,

and in double unresolved limits

$\mathcal{O}_{n+2}^{(j)}\left(p_{1}, \ldots, p_{n+2}\right) \rightarrow \mathcal{O}_{n}^{(j)}\left(p_{1}^{\prime}, \ldots, p_{n}^{\prime}\right)$.

$\mathcal{A}_{n}$ is the amplitude with $n$ partons. At NNLO we need the following expansions of the amplitudes:

$$
\begin{aligned}
& \left|\mathcal{A}_{n}\right|^{2}=\mathcal{A}_{n}^{(0)}{ }^{*} \mathcal{A}_{n}^{(0)}+\left(\mathcal{A}_{n}^{(0)}{ }^{*} \mathcal{A}_{n}^{(1)}+\mathcal{A}_{n}^{(1)}{ }^{*} \mathcal{A}_{n}^{(0)}\right) \\
& \quad+\left(\mathcal{A}_{n}^{(0)}{ }^{*} \mathcal{A}_{n}^{(2)}+\mathcal{A}_{n}^{(2)}{ }^{*} \mathcal{A}_{n}^{(0)}+\mathcal{A}_{n}^{(1)}{ }^{*} \mathcal{A}_{n}^{(1)}\right) \\
& \left|\mathcal{A}_{n+1}\right|^{2}=\mathcal{A}_{n+1}^{(0)}{ }^{*} \mathcal{A}_{n+1}^{(0)} \\
& \quad+\left(\mathcal{A}_{n+1}^{(0)}{ }^{*} \mathcal{A}_{n+1}^{(1)}+\mathcal{A}_{n+1}^{(1)}{ }^{*} \mathcal{A}_{n+1}^{(0)}\right) \\
& \left|\mathcal{A}_{n+2}\right|^{2}=\mathcal{A}_{n+2}^{(0)}{ }^{*} \mathcal{A}_{n+2}^{(0)} .
\end{aligned}
$$

Here $\mathcal{A}_{n}^{(l)}$ denotes an amplitude with $n$ partons and $l$ loops. The master formula eq. (11) is rewrit- 
ten symbolically as

$\left\langle\mathcal{O}^{(j)}\right\rangle=\sum_{n} \int \mathcal{O}_{n}^{(j)} d \sigma_{n}$

and the LO, NLO and NNLO contribution as

$$
\begin{aligned}
&\left\langle\mathcal{O}^{(j)}\right\rangle^{L O}=\int \mathcal{O}_{n}^{(j)} d \sigma_{n}^{(0)}, \\
&\left\langle\mathcal{O}^{(j)}\right\rangle^{N L O}=\int \mathcal{O}_{n+1}^{(j)} d \sigma_{n+1}^{(0)}+\int \mathcal{O}_{n}^{(j)} d \sigma_{n}^{(1)}, \\
&\left\langle\mathcal{O}^{(j)}\right\rangle^{N N L O}=\int \mathcal{O}_{n+2}^{(j)} d \sigma_{n+2}^{(0)} \\
& \quad+\int \mathcal{O}_{n+1}^{(j)} d \sigma_{n+1}^{(1)}+\int \mathcal{O}_{n}^{(j)} d \sigma_{n}^{(2)} .
\end{aligned}
$$

To compute the two-loop amplitudes, new techniques have been developed. Examples include the application of the Mellin-Barnes transformation [23, 24], differential equations [25], nested sums [26-29], or sector decomposition [18]. In addition, several methods, which reduce the workload have been invented. Prominent examples are integration-by-parts-identities [30,31], reduction algorithms $[32,33]$ or the cut technique [34]. In the results, like for example in the two-loop amplitude for $e^{+} e^{-} \rightarrow 3$ jets $[35,36]$, a new class of mathematical functions appears. These are the multiple polylogarithms.

\section{MULTIPLE POLYLOGARITHMS}

The multiple polylogarithms are defined by

$$
\begin{aligned}
& \operatorname{Li}_{m_{1}, \ldots, m_{k}}\left(x_{1}, \ldots, x_{k}\right)= \\
& \sum_{i_{1}>i_{2}>\ldots>i_{k}>0} \frac{x_{1}^{i_{1}}}{i_{1}^{m_{1}}} \ldots \frac{x_{k}^{i_{k}}}{i_{k}^{m_{k}}} .
\end{aligned}
$$

Alternatively, they can be defined by an integral representation. We introduce the auxiliary functions

$$
\begin{aligned}
& G\left(z_{1}, \ldots, z_{k} ; y\right)= \\
& \quad \int_{0}^{y} \frac{d t_{1}}{t_{1}-z_{1}} \int_{0}^{t_{1}} \frac{d t_{2}}{t_{2}-z_{2}} \ldots \int_{0}^{t_{k-1}} \frac{d t_{k}}{t_{k}-z_{k}} .
\end{aligned}
$$

In this definition one variable is redundant due to the following scaling relation:

$G\left(z_{1}, \ldots, z_{k} ; y\right)=G\left(x z_{1}, \ldots, x z_{k} ; x y\right)$
With the short-hand notation

$$
\begin{aligned}
& G_{m_{1}, \ldots, m_{k}}\left(z_{1}, \ldots, z_{k} ; y\right)= \\
& G(\underbrace{0, \ldots, 0}_{m_{1}-1}, z_{1}, \ldots, z_{k-1}, \underbrace{0 \ldots, 0}_{m_{k}-1}, z_{k} ; y)
\end{aligned}
$$

One then finds

$$
\begin{aligned}
& \operatorname{Li}_{m_{1}, \ldots, m_{k}}\left(x_{1}, \ldots, x_{k}\right)= \\
& (-1)^{k} G_{m_{1}, \ldots, m_{k}}\left(\frac{1}{x_{1}}, \frac{1}{x_{1} x_{2}}, \ldots, \frac{1}{x_{1} \ldots x_{k}} ; 1\right) .
\end{aligned}
$$

Multiple polylogarithms have interesting mathematical properties. In particular, there are two Hopf algebra structures defined on them [36-38]. At the end of the day of an analytic calculation physicists would like to get a number. This requires a method for the numerical evaluation of multiple polylogarithms. The simplest example is the numerical evaluation of the dilogarithm [39]:

$\operatorname{Li}_{2}(x)=-\int_{0}^{x} d t \frac{\ln (1-t)}{t}=\sum_{n=1}^{\infty} \frac{x^{n}}{n^{2}}$

The power series expansion can be evaluated numerically, provided $|x|<1$. Using the functional equations

$$
\begin{aligned}
& \mathrm{Li}_{2}(x)=-\mathrm{Li}_{2}\left(\frac{1}{x}\right)-\frac{\pi^{2}}{6}-\frac{1}{2}(\ln (-x))^{2}, \\
& \mathrm{Li}_{2}(x)=-\mathrm{Li}_{2}(1-x)+\frac{\pi^{2}}{6}-\ln (x) \ln (1-x),
\end{aligned}
$$

any argument of the dilogarithm can be mapped into the region $|x| \leq 1$ and $-1 \leq \operatorname{Re}(x) \leq 1 / 2$. The numerical computation can be accelerated by using an expansion in $[-\ln (1-x)]$ and the Bernoulli numbers $B_{i}$ :

$\mathrm{Li}_{2}(x)=\sum_{i=0}^{\infty} \frac{B_{i}}{(i+1) !}(-\ln (1-x))^{i+1}$.

The generalisation to multiple polylogarithms proceeds along the same lines [40]: Using the integral representation of

$G_{m_{1}, \ldots, m_{k}}\left(z_{1}, z_{2}, \ldots, z_{k} ; y\right)$

one transforms all arguments into a region, where one has a converging power series expansion. To accelerate the convergence of the series the Hölder convolution is used [41]. 


\section{INFRA-RED DIVERGENCES}

The individual contributions on the r.h.s. of eq. (6) to $\left\langle\mathcal{O}^{(j)}\right\rangle^{N L O}$ and $\left\langle\mathcal{O}^{(j)}\right\rangle^{N N L O}$ are in general infra-red divergent, only the sum is finite. However, these contributions live on different phase spaces, which prevents a naive Monte Carlo approach. To render the individual contributions finite, one adds and subtracts suitable chosen terms. The NLO contribution is given by

$$
\begin{gathered}
\left\langle\mathcal{O}^{(j)}\right\rangle^{N L O}=\int\left(\mathcal{O}_{n+1}^{(j)} d \sigma_{n+1}^{(0)}-\mathcal{O}_{n}^{(j)} \circ d \alpha_{n}^{(0,1)}\right) \\
+\int\left(\mathcal{O}_{n}^{(j)} d \sigma_{n}^{(1)}+\mathcal{O}_{n}^{(j)} \circ d \alpha_{n}^{(0,1)}\right) .
\end{gathered}
$$

The notation $\mathcal{O}_{n}^{(j)} \circ d \alpha_{n}^{(0,1)}$ is a reminder, that in general the approximation is a sum of terms

$\mathcal{O}_{n}^{(j)} \circ d \alpha_{n}^{(0,1)}=\sum \mathcal{O}_{n}^{(j)} d \alpha_{n}^{(0,1)}$

and the mapping used to relate the $n+1$ parton configuration to a $n$ parton configuration differs in general for each summand. In a similar way, the NNLO contribution is written as

$$
\begin{aligned}
\left\langle\mathcal{O}^{(j)}\right\rangle^{N N L O}= & \left\langle\mathcal{O}^{(j)}\right\rangle_{n+2}^{N N L O}+\left\langle\mathcal{O}^{(j)}\right\rangle_{n+1}^{N N L O} \\
& +\left\langle\mathcal{O}^{(j)}\right\rangle_{n}^{N N L O}
\end{aligned}
$$

with

$$
\begin{aligned}
\left\langle\mathcal{O}^{(j)}\right\rangle_{n+2}^{N N L O} & =\int\left(\mathcal{O}_{n+2}^{(j)} d \sigma_{n+2}^{(0)}-\mathcal{O}_{n+1}^{(j)} \circ d \alpha_{n+1}^{(0,1)}\right. \\
\left.-\mathcal{O}_{n}^{(j)} \circ d \alpha_{n}^{(0,2)}\right), & \\
\left\langle\mathcal{O}^{(j)}\right\rangle_{n+1}^{N N L O} & =\int\left(\mathcal{O}_{n+1}^{(j)} d \sigma_{n+1}^{(1)}+\mathcal{O}_{n+1}^{(j)} \circ d \alpha_{n+1}^{(0,1)}\right. \\
\left.-\mathcal{O}_{n}^{(j)} \circ d \alpha_{n}^{(1,1)}\right), & \\
\left\langle\mathcal{O}^{(j)}\right\rangle_{n}^{N N L O} & =\int\left(\mathcal{O}_{n}^{(j)} d \sigma_{n}^{(2)}+\mathcal{O}_{n}^{(j)} \circ d \alpha_{n}^{(0,2)}\right. \\
+ & \left.\mathcal{O}_{n}^{(j)} \circ d \alpha_{n}^{(1,1)}\right) .
\end{aligned}
$$

$d \alpha_{n+1}^{(0,1)}$ is the NLO subtraction term for $(n+1)$ parton configurations, $d \alpha_{n}^{(0,2)}$ and $d \alpha_{n}^{(1,1)}$ are generic NNLO subtraction terms. It is convenient to split these terms into

$$
\begin{aligned}
& d \alpha_{n}^{(0,2)}=d \alpha_{(0,0), n}^{(0,2)}-d \alpha_{(0,1), n}^{(0,2)} \\
& d \alpha_{n}^{(1,1)}=d \alpha_{(1,0), n}^{(1,1)}+d \alpha_{(0,1), n}^{(1,1)}
\end{aligned}
$$

such that $d \alpha_{(0,0), n}^{(0,2)}$ and $d \alpha_{(1,0), n}^{(1,1)}$ approximate $d \sigma_{n+2}^{(0)}$ and $d \sigma_{n+1}^{(1)}$, respectively. $d \alpha_{(0,1), n}^{(0,2)}$ and $d \alpha_{(0,1), n}^{(1,1)}$ are approximations to $d \alpha_{n+1}^{(0,1)}$.

\section{PHASE SPACE GENERATION}

It is a well-known fact, that in the collinear limit spin correlations remain. For example, the spin-dependent splitting functions for $g \rightarrow q \bar{q}$ reads

$P_{g \rightarrow q \bar{q}}^{(0,1)}=\frac{2}{s_{i j}}\left[-g^{\mu \nu}+4 z(1-z) \frac{k_{\perp}^{\mu} k_{\perp}^{\nu}}{k_{\perp}^{2}}\right]$.

The collinear limits occurs for $k_{\perp}^{2} \rightarrow 0$. The term

$\mathcal{A}_{\mu} \frac{1}{s_{i j}} \frac{k_{\perp}^{\mu} k_{\perp}^{\nu}}{k_{\perp}^{2}} \mathcal{A}_{\nu}$

is proportional to the spin correlation. In four dimensions the spin-averaged splitting functions are obtained by integrating over the azimuthal angle $\varphi$ of $p_{i}$ around $p$. By using spin-averaged antenna functions, the subtraction terms have not the same point-wise singular behaviour as the matrix elements, which is required for local subtraction terms. Instead, cancellations of singularities occurs only after an integration over the azimuthal angle over all collinear splittings of the matrix elements. For $n$ final-state particles, this is a one-dimensional integration in the $(3 n-4)$ dimensional phase space. It can be shown, that in the single collinear limit, the spin correlation depends on the azimuthal angle $\varphi$ as

$\mathcal{A}_{\mu} \frac{1}{s_{i j}} \frac{k_{\perp}^{\mu} k_{\perp}^{\nu}}{k_{\perp}^{2}} \mathcal{A}_{\nu} \sim C_{0}+C_{2} \cos (2 \varphi+\alpha)$.

One can therefore perform the average with two points, where the azimuthal angle takes the values $\varphi$ and $\varphi+\pi / 2$, while all other coordinates remain fixed. In detail this is done as follows: We partition the phase space into different channels. Within one channel, the phase space is generated iteratively according to

$d \phi_{n+1}=d \phi_{n} d \phi_{\text {Dipole } i, j, k}$.

For each channel we require that the product $s_{i j} s_{j k}$ is the smallest among all considered channels and that $s_{i j}<s_{j k}$. Therefore it follows that 
with channel $(i, j, k)$ also channel $(k, j, i)$ has to be included into the partioning of the phase space. For the dipole phase space measure we have

$d \phi_{\text {dipole }}=\frac{s_{i j k}}{32 \pi^{3}} \int_{0}^{1} d y(1-y) \int_{0}^{1} d z \int_{0}^{2 \pi} d \varphi$.

We can therefore generate the $(n+1)$-parton configuration from the $n$-parton configuration by using three random numbers $u_{1}, u_{2}, u_{3}$ and by setting

$y=u_{1}, \quad z=u_{2} \quad \varphi=2 \pi u_{3}$.

This defines the invariants as

$$
\begin{aligned}
& s_{i j}=y s_{i j k}, \quad s_{i k}=z(1-y) s_{i j k}, \\
& s_{j k}=(1-z)(1-y) s_{i j k} .
\end{aligned}
$$

From these invariants and the value of $\varphi$ we can reconstruct the four-momenta of the $(n+1)$ parton configuration [42]. The additional phase space weight due to the insertion of the $(n+1)$-th particle is

$w=\frac{s_{i j k}}{16 \pi^{2}}(1-y)$.

We have therefore a parameterization of the phase space, such that for every collinear limit the azimuthal average can be easily performed, while keeping all other coordinates fixed. It is clear that this procedure can be iterated for multiple collinear emissions.

\section{NUMERICAL RESULTS}

A test case for the methods discussed above for the cancellation of infra-red divergences and numerical phase space integration is the process $e^{+} e^{-} \rightarrow 2$ jets. The two-jet cross section has the perturbative expansion

$$
\begin{aligned}
& \langle\sigma\rangle^{(2-j e t)}= \\
& \langle\sigma\rangle^{(0)}\left(1+\frac{\alpha_{s}}{2 \pi} B^{(2-j e t)}+\left(\frac{\alpha_{s}}{2 \pi}\right)^{2} C^{(2-j e t)}\right) .
\end{aligned}
$$

$\langle\sigma\rangle^{(0)}$ is the total hadronic cross section at leading order, the numerical value at LEP energies $\sqrt{Q^{2}}=91.187 \mathrm{GeV}$ is $\langle\sigma\rangle^{(0)}=40807.4 \mathrm{pb}$. The jets are defined according to the Durham jet algorithm with $y=0.01$. For the numerical values of the NLO and NNLO coefficients $B^{(2-j e t)}$ and $C^{(2-j e t)}$ one obtains

$B^{(2-j e t)}=-13.674 \pm 0.004$,
$C^{(2-j e t)}=-231.6 \pm 0.3$.

The correctness of this result can be verified with the help of the known results for the total hadronic cross section at NNLO, the three-jet cross section at NLO and the four-jet cross section at $\mathrm{LO}$. We have the perturbative expansions

$$
\begin{aligned}
& \langle\sigma\rangle^{(t o t)}= \\
& \langle\sigma\rangle^{(0)}\left(1+\frac{\alpha_{s}}{2 \pi} B^{(t o t)}+\left(\frac{\alpha_{s}}{2 \pi}\right)^{2} C^{(t o t)}\right), \\
& \langle\sigma\rangle^{(3-j e t)}= \\
& \langle\sigma\rangle^{(0)}\left(\frac{\alpha_{s}}{2 \pi} B^{(3-j e t)}+\left(\frac{\alpha_{s}}{2 \pi}\right)^{2} C^{(3-j e t)}\right), \\
& \langle\sigma\rangle^{(4-j e t)}=\langle\sigma\rangle^{(0)}\left(\frac{\alpha_{s}}{2 \pi}\right)^{2} C^{(4-j e t)} .
\end{aligned}
$$

The values of the coefficients are:

$$
\begin{aligned}
& B^{(t o t)}=2, C^{(t o t)}=5.64, \\
& B^{(3-j e t)}=15.679 \pm 0.004 \\
& C^{(3-j e t)}=153.2 \pm 0.4, C^{(4-j e t)}=84.39 \pm 0.05
\end{aligned}
$$

We must have

$$
\begin{aligned}
& B^{(t o t)}=B^{(2-j e t)}+B^{(3-j e t)}, \\
& C^{(t o t)}=C^{(2-j e t)}+C^{(3-j e t)}+C^{(4-j e t)} .
\end{aligned}
$$

We find

$$
\begin{aligned}
& B^{(2-j e t)}+B^{(3-j e t)}=2.005 \pm 0.006, \\
& C^{(2-j e t)}+C^{(3-j e t)}+C^{(4-j e t)}=6.0 \pm 0.5,
\end{aligned}
$$

which agrees well with the values of $B^{(t o t)}$ and $C^{(t o t)}$

\section{OUTLOOK}

Fully differential numerical programs at NNLO are a challenging task. I reviewed the state of the art and discussed the calculation of two-loop amplitudes, the cancellation of infra-red divergences and methods for an efficient generation of the phase space. As a first application I presented complete results for the NNLO correction to the process $e^{+} e^{-} \rightarrow 2$ jets, based on the subtraction 
method. This gives us confidence, that the extension to other processes like $e^{+} e^{-} \rightarrow 3$ jets or $p p \rightarrow 2$ jets is within reach.

\section{REFERENCES}

1. M. Dobbs et al., (2004), hep-ph/0403100

2. D.A. Kosower, Phys. Rev. D67 (2003) 116003, hep-ph/0212097.

3. D.A. Kosower, Phys. Rev. Lett. 91 (2003) 061602, hep-ph/0301069,

4. S. Weinzierl, JHEP 03 (2003) 062, hep-ph/0302180

5. S. Weinzierl, JHEP 07 (2003) 052, hep-ph/0306248,

6. C. Anastasiou, K. Melnikov and F. Petriello, Phys. Rev. D69 (2004) 076010, hep-ph/0311311.

7. A. Gehrmann-De Ridder, T. Gehrmann and E.W.N. Glover, JHEP 09 (2005) 056, hep-ph/0505111,

8. T. Binoth and G. Heinrich, Nucl. Phys. B693 (2004) 134, hep-ph/0402265

9. G. Heinrich, (2006), hep-ph/0601062

10. W.B. Kilgore, Phys. Rev. D70 (2004) 031501, hep-ph/0403128,

11. S. Frixione and M. Grazzini, JHEP 06 (2005) 010, hep-ph/0411399

12. G. Somogyi, Z. Trocsanyi and V. Del Duca, JHEP 06 (2005) 024, hep-ph/0502226

13. S. Frixione, Z. Kunszt and A. Signer, Nucl. Phys. B467 (1996) 399, hep-ph/9512328,

14. S. Catani and M.H. Seymour, Nucl. Phys. B485 (1997) 291, hep-ph/9605323

15. S. Dittmaier, Nucl. Phys. B565 (2000) 69, hep-ph/9904440,

16. L. Phaf and S. Weinzierl, JHEP 04 (2001) 006, hep-ph/0102207

17. S. Catani et al., Nucl. Phys. B627 (2002) 189, hep-ph/0201036.

18. T. Binoth and G. Heinrich, Nucl. Phys. B585 (2000) 741, hep-ph/0004013

19. C. Anastasiou, K. Melnikov and F. Petriello, Phys. Rev. Lett. 93 (2004) 032002, hep-ph/0402280,

20. C. Anastasiou, K. Melnikov and F. Petriello, Phys. Rev. Lett. 93 (2004) 262002, hep-ph/0409088,
21. C. Anastasiou, K. Melnikov and F. Petriello, Nucl. Phys. B724 (2005) 197, hep-ph/0501130

22. S. Weinzierl, (2006), hep-ph/0606008

23. V.A. Smirnov, Phys. Lett. B460 (1999) 397, hep-ph/9905323

24. I. Bierenbaum and S. Weinzierl, Eur. Phys. J. C32 (2003) 67, hep-ph/0308311

25. T. Gehrmann and E. Remiddi, Nucl. Phys. B580 (2000) 485, hep-ph/9912329

26. S. Moch, P. Uwer and S. Weinzierl, J. Math. Phys. 43 (2002) 3363, hep-ph/0110083,

27. S. Weinzierl, Comput. Phys. Commun. 145 (2002) 357, math-ph/0201011

28. S. Weinzierl, J. Math. Phys. 45 (2004) 2656, hep-ph/0402131.

29. S. Moch and P. Uwer, Comput. Phys. Commun. 174 (2006) 759, math-ph/0508008

30. K.G. Chetyrkin and F.V. Tkachov, Nucl. Phys. B192 (1981) 159,

31. F.V. Tkachov, Phys. Lett. B100 (1981) 65,

32. O.V. Tarasov, Phys. Rev. D54 (1996) 6479, hep-th/9606018

33. S. Laporta, Int. J. Mod. Phys. A15 (2000) 5087, hep-ph/0102033

34. Z. Bern, L. Dixon and D.A. Kosower, JHEP 01 (2000) 027, hep-ph/0001001

35. L.W. Garland et al., Nucl. Phys. B642 (2002) 227, hep-ph/0206067.

36. S. Moch, P. Uwer and S. Weinzierl, Phys. Rev. D66 (2002) 114001, hep-ph/0207043

37. S. Weinzierl, (2003), hep-th/0305260

38. S. Weinzierl, (2006), hep-ph/0604068

39. G. 't Hooft and M.J.G. Veltman, Nucl. Phys. B153 (1979) 365,

40. J. Vollinga and S. Weinzierl, Comput. Phys. Commun. 167 (2005) 177, hep-ph/0410259

41. J.M. Borwein et al., Trans. Amer. Math. Soc. 353:3 (2001) 907, math.CA/9910045

42. S. Weinzierl and D.A. Kosower, Phys. Rev. D60 (1999) 054028, hep-ph/9901277. 